\title{
Radiation-Recall Dermatitis
}

National Cancer Institute

\section{Source}

National Cancer Institute. Radiation-Recall Dermatitis. NCI Thesaurus. Code C78580.

Acute skin inflammatory reaction caused by drugs, especially chemotherapeutic agents, weeks or months following radiotherapy. The inflammatory reaction is confined to the previously irradiated skin and the symptoms disappear after the removal of the pharmacologic agent. 